\title{
Bias-controlled ultrafast demagnetization in magnetic tunnel junctions
}

\author{
M. Savoini, ${ }^{1, *}$ C. Piovera,${ }^{1,2}$ C. Rinaldi, ${ }^{3}$ E. Albisetti, ${ }^{3}$ D. Petti, ${ }^{3}$ A. R. Khorsand,${ }^{1}$ L. Duò, ${ }^{2}$ C. Dallera, ${ }^{2}$ M. Cantoni, ${ }^{3}$ \\ R. Bertacco, ${ }^{3}$ M. Finazzi, ${ }^{2}$ E. Carpene, ${ }^{4}$ A. V. Kimel, ${ }^{1}$ A. Kirilyuk, ${ }^{1}$ and Th. Rasing ${ }^{1}$ \\ ${ }^{1}$ Radboud University Nijmegen, Institute for Molecules and Materials, Heyendaalseweg 135, Nijmegen, The Netherlands \\ ${ }^{2}$ CNISM-Dipartimento di Fisica, Politecnico di Milano, Piazza Leonardo da Vinci 32, 20133 Milan, Italy \\ ${ }^{3}$ LNESS-Dipartimento di Fisica del Politecnico di Milano, Via Anzani 42, 22100 Como, Italy \\ ${ }^{4}$ IFN-CNR, Dipartimento di Fisica, Politecnico di Milano, Piazza Leonardo da Vinci 32, 20133 Milan, Italy
}

(Received 31 July 2013; revised manuscript received 28 November 2013; published 4 April 2014)

\begin{abstract}
We report on the possibility of controlling the maximum demagnetization induced by a femtosecond laser pulse in a CoFeB-based magnetic tunnel junction, by tuning the external bias applied to the junction. We explain this effect in terms of laser-induced spin-polarized currents which can be controlled by tuning the conductivity of the junction. This demonstrates the contribution of spin-polarized currents for laser-induced demagnetization and may pave the way to increase the speed of spintronic devices, using ultrashort laser pulses.
\end{abstract}

PACS number(s): 75.40.Gb, 72.15.Gd, 75.78.Jp, 85.70.Sq

Spin-polarized transport [1,2] and laser-induced ultrafast magnetic phenomena [3,4] are strongly, but separately, developing areas in condensed matter science, attracting an ever expanding interest from the scientific community for both fundamental and technological reasons. Despite their common denominator of spin control, only recently new possibilities to manipulate spins inside spintronic devices such as a magnetic tunnel junction (MTJ) were presented [5-9], leading to the discovery of the magneto-Seebeck effect [7-9]. Alternatively, the magneto-optical Kerr effect (MOKE) has been applied to observe the magnetization dynamics in magnetic double layers excited by spin-polarized currents [10-12]. Moreover, whereas the potential relevance of spin-polarized electron transport in laser-induced demagnetization was pointed out [13], a clear experimental confirmation with a quantitative measure of its relevance is still lacking.

In this Rapid Communication we present our results concerning laser-induced ultrafast demagnetization in a biased MTJ, showing that we can control the magnetization dynamics with an external bias, exploiting the spin filtering properties of MTJs. After excluding all possible spurious contributions, we can explain this ultrafast demagnetization in MTJs in terms of photoexcited currents promoted across the tunnel barrier. This implies that, in a multilayer system, a contribution (up to about $40 \%$ ) to ultrafast demagnetization may be due to transport phenomena. Our results imply the possibility of optically addressed spintronic devices which may benefit in speed of operation and performances compared to their all-electric analogs [14,15].

The studied MTJ samples are heterostructures of (from bottom to top, thickness reported in parentheses in nanometers): $\operatorname{Ir}_{22} \mathrm{Mn}_{78}(15) / \mathrm{Co}_{40} \mathrm{Fe}_{40} \mathrm{~B}_{20}(5) / \mathrm{MgO}(2) /$ $\mathrm{Co}_{40} \mathrm{Fe}_{40} \mathrm{~B}_{20}(10)$. These layers are grown on a buffer stack of $\mathrm{Si} / \mathrm{SiO}_{2}(1000) / \mathrm{Ta}(5) / \mathrm{Ru}(18) / \mathrm{Ta}(3)$. The devices are then capped with $\mathrm{MgO}(2) / \mathrm{SiO}_{2}(2)$. Transparent materials $\left(\mathrm{MgO} / \mathrm{SiO}_{2}\right)$ were used as a top coating to have direct optical access to the magnetic layers. Also the geometry of the gold contacts has been changed accordingly. Moreover, the topmost $\mathrm{Co}_{40} \mathrm{Fe}_{40} \mathrm{~B}_{20}$ magnetic layer has a larger thickness than the

\footnotetext{
*m.savoini@science.ru.nl
}

buried one. We dimensioned these layers in order to optimize the optical absorption in the top layer and to minimize the laser power absorbed by the buried $\mathrm{CoFeB}$ and $\mathrm{IrMn}$ layers. A three-dimensional (3D) sketch of the device is presented in the inset of Fig. 1(b). Extensive details in sample preparation and device geometry are presented in the Supplemental Material [16].

The static (no optical pumping) magneto-optical and electric characterization of one of these devices with diameter $d=125 \mu \mathrm{m}$ are presented in Fig. 1. Figure 1(a) shows (in full diamonds) that the Kerr ellipticity signal $\epsilon$, proportional to the magnetization, is composed of two contributions: a squared loop around zero field, characterized by a small coercivity associated with the top (free) layer, and a broader and smaller one shifted by exchange bias, corresponding to the buried (pinned) layer. The resistance of the device [red open squares in Fig. 1(a)], measured with an external voltage applied between the two magnetic layers of $20 \mathrm{mV}$, presents two states with low (high) resistance corresponding to parallel (antiparallel) alignment of the magnetizations of the two $\mathrm{CoFeB}$ layers. The corresponding tunneling magnetoresistance (TMR), defined as $\mathrm{TMR}=\left(R_{\downarrow \uparrow}-R_{\uparrow \uparrow}\right) / R_{\uparrow \uparrow}$, is about $10 \%$ at room temperature (RT) for the device reported in Fig. 1(a). All the measured devices presented typical TMR values between $8 \%$ and $40 \%$ at RT, with larger values generally found in smaller devices. The geometry of the contacts and the direct growth of $\mathrm{MgO}$ on top of the $\mathrm{FeCoB}$ layer (which reduces the B interdiffusion within the ferromagnetic layer, [17]) contribute in reducing the maximum measured TMR values, as compared with standard devices $[15,18]$. Nevertheless, the choice of an insulator such as $\mathrm{MgO}$ as a capping layer has been dictated by the necessity to avoid the extra currents that a metallic cover layer such as Ta would inject into the device stack upon illumination, thus hindering the interpretation of the collected data presented in the following of this Rapid Communication. Figure 1(b) presents the TMR value as a function of the bias voltage $V_{\text {bias }}$, showing an $\sim 30 \%$ TMR reduction by increasing $\left|V_{\text {bias }}\right|$ from 0 to $400 \mathrm{mV}$, independently from the sign of $V_{\text {bias }}$ [19]. As detailed further below, it is important to exclude artifacts that might result from the magneto-optical properties of the layers depending on the external bias. Thus, we have acquired also hysteresis loops as a function of the bias voltage 

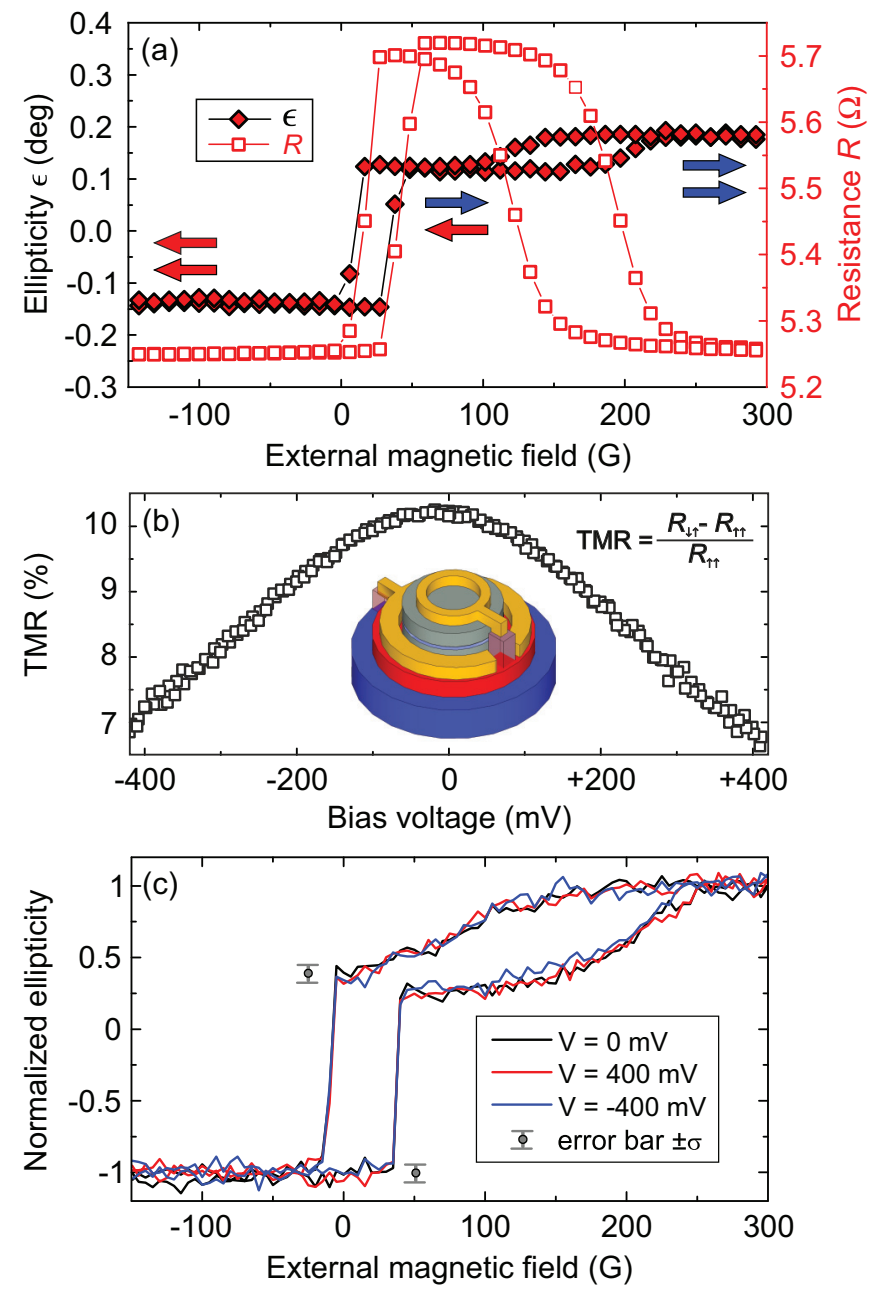

FIG. 1. (Color online) (a) Static Kerr ellipticity $\epsilon$ for an exchange-biased tunneling junction and electrical resistance $R$ as a function of the external magnetic field. The arrows indicate the relative magnetic coupling of the $\mathrm{CoFeB}$ layers as a function of the external magnetic fields. (b) TMR value as a function of the external bias voltage applied to the structure. Inset: 3D representation of the layer and contact geometry. The color coding is reported in the Supplemental Material [16]. (c) Typical bias-dependent hysteresis loop of the magnetic tunnel junctions; no apparent dependence is measurable within the experimental noise. The curves are measured on a device with diameter $d=125 \mu \mathrm{m}$.

$(-400,0,+400 \mathrm{mV})$ showing that, within our experimental noise, no variation is measurable, as presented in Fig. 1(c).

The laser-induced magnetization dynamics was studied by a time-resolved stroboscopic magneto-optical pump-probe technique at room temperature, using an amplified Ti:sapphire system (repetition rate $f=250 \mathrm{kHz}$, output power $P_{a v}=1.5 \mathrm{~W}$, emission wavelength $\lambda=800 \mathrm{~nm}$, pulse duration $\delta=80 \mathrm{fs}$ ). The laser beam was split with a $90 \%-10 \%$ beam splitter to set up a noncollinear pump-probe scheme. The pump beam was doubled in frequency with a nonlinear Barium Boron Oxide crystal. Both beams, after being delayed with respect to each other, were linearly polarized ( $s$ polarization for the pump, $p$ polarization for the probe) and then superimposed on the sample. To study the MTJs (displaying in-plane magnetic anisotropy), the probe beam incidence angle was set equal to $45^{\circ}$ to maximize the sensitivity to the Kerr ellipticity in the longitudinal MOKE configuration [20]. The pump beam impinged onto the sample with an angle roughly equal to $60^{\circ}$ with respect to the surface normal and was $s$ polarized. This geometry ensured a sizable reflection from the dielectric coating and limited the penetration of the pump beam mainly to the top magnetic layer. We estimate that $72 \%$ of the impinging pump fluence is reflected, $18 \%$ is absorbed by the free layer, $4 \%$ by the pinned one, and another $5 \%$ by the IrMn underlayer. The remaining laser power is absorbed in the buffer layers [16]. As presented in [16], these absorbed intensities correspond to an average temperature increase of about $\Delta T=250 \mathrm{~K}$ in the free layer, $100 \mathrm{~K}$ in the pinned, and $50 \mathrm{~K}$ in the IrMn one. After interacting with the sample, the reflected probe beam crosses a quarter-wave plate, before impinging on a balanced detection scheme.

The laser-induced changes in magnetization are studied by recording the variation of Kerr ellipticity $\epsilon$ experienced by the probe beam as a function of the relative time delay $t$ between pump and probe. By measuring the sample response at different external magnetic fields, and thus at different relative orientations of the layer magnetizations, we are able to evaluate the magnetization-dependent variations in $\epsilon$ for different cases, namely,

$$
\begin{gathered}
\frac{\Delta \epsilon^{\mathrm{top}}(t)}{\epsilon(0)} \propto\left[\Delta \epsilon_{\uparrow \uparrow}(t)-\Delta \epsilon_{\downarrow \uparrow}(t)\right] / \epsilon(0), \\
\frac{\Delta \epsilon^{\text {total }}(t)}{\epsilon(0)} \propto\left[\Delta \epsilon_{\uparrow \uparrow}(t)-\Delta \epsilon_{\downarrow \downarrow}(t)\right] / \epsilon(0),
\end{gathered}
$$

with $\Delta \epsilon^{\text {top }}(t)$ the pump-induced variation in $\epsilon$ solely in the top layer. Extrapolating the $\Delta \epsilon^{\text {top }}(t)$ is possible thanks to the exchange bias provided by the IrMn, which pins the buried ferromagnetic layer. $\Delta \epsilon^{\text {top }}(t)$ can thus be measured by reversing the top layer magnetization with the external magnetic field while keeping the bottom one unchanged. $\Delta \epsilon^{\text {total }}(t)$ is the pump-induced variation for the whole device, $\epsilon(0)=\epsilon^{\text {total }}(0)$ is the static ellipticity reported in Fig. 1(a), and $\Delta \epsilon_{\uparrow \uparrow(\downarrow \uparrow, \downarrow \downarrow)}(t)$ is the measured change corresponding to the layers coupling indicated by the orientation of the arrows.

Figure 2(a) shows the ultrafast laser-induced changes in $\Delta \epsilon^{\text {top }} / \epsilon(0)$ as a function of the pump-probe delay and for different bias voltages. We limited our study to $\left|V_{\text {bias }}\right|<$ $500 \mathrm{mV}$ to avoid the breakdown of the $\mathrm{MgO}$ barrier. The measurements were performed at three different external magnetic fields: $H_{\text {ext }}=-100,+75$, and $+300 \mathrm{G}$, to set the coupling in the parallel, antiparallel, and reversed parallel states. The fluence on the sample was $4.4 \pm 0.3 \mathrm{~mJ} / \mathrm{cm}^{2}$. In the case of unbiased measurements, an ultrafast change in ellipticity is triggered with a signal reduction of $11.5 \%$ within 380 fs. A remarkable finding is that we are able to strongly modulate the $\Delta \epsilon^{\mathrm{top}}(t)$ signal by applying a bias [see Fig. 2(a)]: $\Delta \epsilon_{\max }^{\text {top }} / \epsilon(0)$, the maximum $\Delta \epsilon^{\text {top }} / \epsilon(0)$ change, is only $8.5 \%$ for $V_{\text {bias }}= \pm 400 \mathrm{mV}$, corresponding to a variation of about $30 \%$ with respect to the zero-bias case. The change in the reflectivity signal at different applied voltages $V_{\text {bias }}$ is shown in the inset of Fig. 2(a). No apparent difference is present within the experimental noise.

Figure 2(b) presents the laser-induced $\Delta \epsilon_{\max }^{\text {top }} / \epsilon(0)$ and $\Delta \epsilon_{\max }^{\text {total }} / \epsilon(0)$ as a function of $V_{\text {bias }}$. At $\left|V_{\text {bias }}\right|$ larger than a 

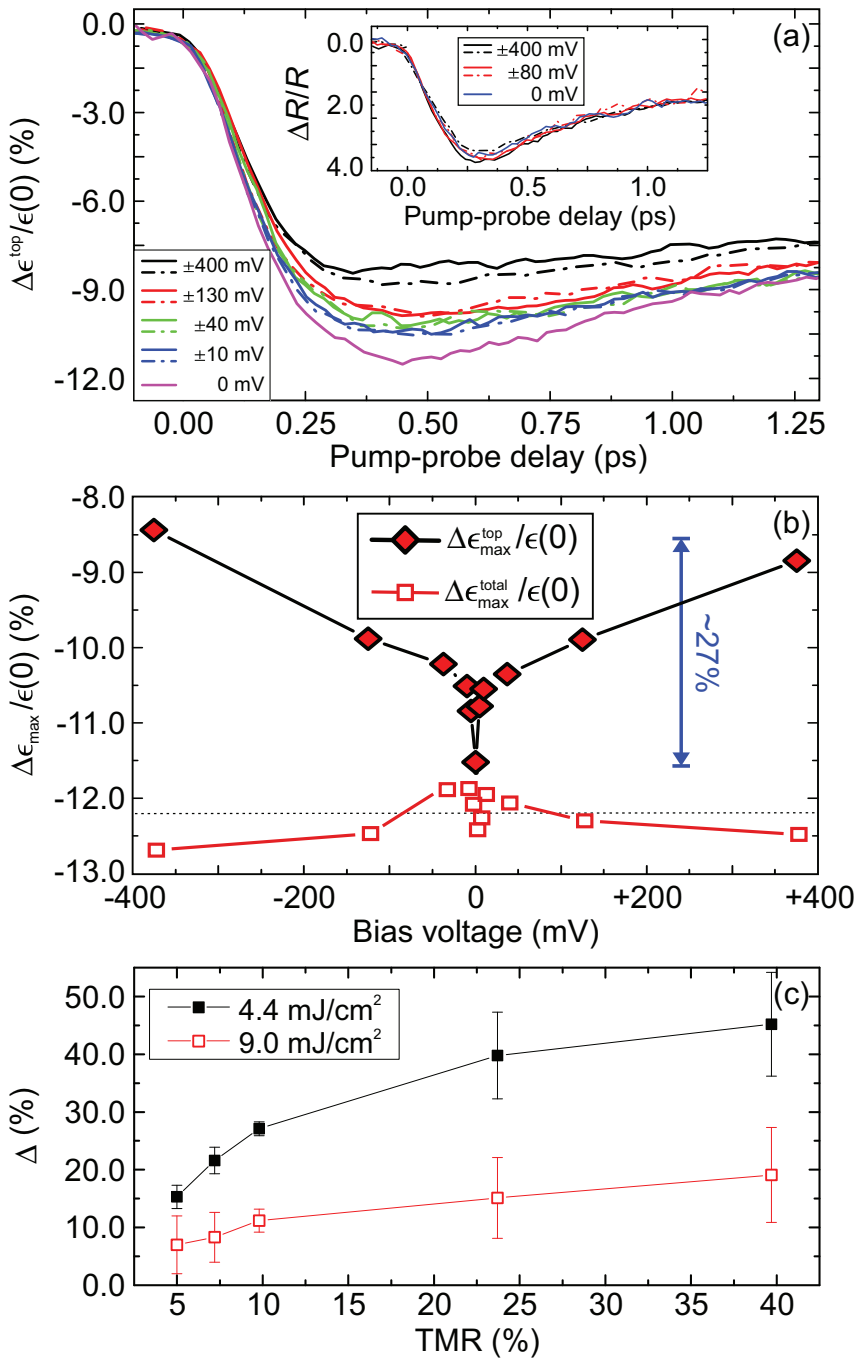

FIG. 2. (Color online) (a) Ultrafast variations in the probe beam ellipticity, measured with longitudinal MOKE as a function of the pump-probe delay and the bias voltage. The curves address the changes in the top layer. The fluence impinging on the sample is $4.4 \mathrm{~mJ} / \mathrm{cm}^{2}$. Inset: ultrafast change in the sample reflectivity as a function of the bias voltage; no apparent variation is measurable. (b) Maximum change in ellipticity as a function of the bias and of the relative orientations of the layer magnetizations [see Eqs. 1(a) and 1(b)]. (c) Maximum demagnetization modulation $\Delta$ achieved in different devices as a function of the corresponding TMR value, at two different fluencies: 4.4 and $9 \mathrm{~mJ} / \mathrm{cm}^{2}$. The curves in (a) and (b) are measured on a device with diameter $d=125 \mu \mathrm{m}$.

few $\mathrm{mV}$ the dependence of $\Delta \epsilon_{\max }^{\text {top }}$ upon $V_{\text {bias }}$ is practically linear. Remarkably, we observe a cusplike behavior around zero $V_{\text {bias }}$, similar to the so-called zero-bias anomaly, measured for similar structures at low temperatures with electrictransport methods [21]. A deeper investigation is necessary to understand the nature of this effect that is measured with optical methods and at room temperature. Figure 2(b) also presents the data extrapolated from $\Delta \epsilon^{\text {total }}(t) / \epsilon(0)$ [16]. First of all, $\left|\Delta \epsilon_{\max }^{\text {total }} / \epsilon(0)\right|$ value at zero bias is about $1 \%$ larger than $\left|\Delta \epsilon_{\max }^{\text {top }} / \epsilon(0)\right|$. This increase can be related to the small laser-induced ellipticity change of the buried layers.
We are therefore quite confident that the optical pump does not strongly affect the pinned $\mathrm{CoFeB}$ and the IrMn layers. However, the voltage dependence of $\Delta \epsilon_{\max }^{\text {total }}\left(V_{\text {bias }}\right)$ shows a very different trend from that of $\Delta \epsilon_{\max }^{\text {top }}\left(V_{\text {bias }}\right): \Delta \epsilon_{\max }^{\text {total }} / \epsilon(0)$ is always in the range $12.2 \pm 0.5 \%$ and does not show a strong dependence on $V_{\text {bias }}$. This different behavior cannot simply be explained by the contribution of the pinned layer, in view of its small demagnetization.

These measurements were also performed on devices with different size. We found that $\Delta \epsilon / \epsilon(0)$ follows the same qualitative bias dependence, with larger MTJs generally presenting lower TMR values and smaller voltagedependent demagnetizations. Smaller devices offered higher TMR values, but the optical measurements were affected by a lower signal-to-noise ratio due to the large amount of scattered light by the ring Au contacts. The results are summarized in Fig. 2(c). Here the maximum modulation $\Delta=$ $\left[\Delta \epsilon_{\max }^{\text {top }}(400 \mathrm{mV})-\Delta \epsilon_{\max }^{\text {top }}(0 \mathrm{mV})\right] / \Delta \epsilon_{\max }^{\text {top }}(0 \mathrm{mV})$ is plotted as a function of the TMR value measured in the corresponding device. By repeating the measurements at higher fluencies, up to $40 \%$ demagnetizations were obtained, but the bias-induced modulation was significantly reduced [red dots in Fig. 2(c)]. This may suggest that ultrafast demagnetization is a combination of local magnetization reduction due to heating (the main contribution) [22-28] and transport phenomena [13,29].

In the following we will present clear arguments to ascribe these observations directly to changes in the magnetization of the free layer, including a contribution from spin-polarized currents.

The Kerr ellipticity $\epsilon$ is a complex function of the material refractive index $\tilde{n}$ and the sample magnetization $M$ [30]. Therefore, in order to relate $\Delta \epsilon$ to real changes in the magnetization $\Delta M$, we have to exclude any possible modulation of the magnetic susceptibility $\chi$ or $\tilde{n}$ induced either by the light or by the bias voltage.

To this purpose, we first excluded possible changes in $\tilde{n}$. By measuring pump-probe reflectivity curves at different bias, we ensured that no bias dependence of $\tilde{n}$ is present [see inset of Fig. 2(a)]. Secondly, we performed careful static MOKE measurements of the hysteresis loops in different devices, as a function of $V_{\text {bias. }}$. We did not observe any dependence (within the experimental noise) of the measured signal, neither in the antiparallel nor in the parallel configuration [see Fig. 1(c)]. This implies that neither $\tilde{n}$ nor $\chi$ depend on $V_{\text {bias }}$ and on the conductivity of the device. This already excludes that the bias voltage might be the source of artifacts that could be interpreted as magnetic effects. Moreover, the bias does not change the absolute magnetization of the sample. Even spin-torque effects are negligible [31-33], as demonstrated in the Supplemental Material. A further possible source of artifacts might be a pump-induced change in the IrMn pinning properties stemming from an increase of the layer temperature rise. To exclude this possibility, hysteresis cycles measured in the absence of the optical pump have been compared with those recorded at $300 \mathrm{fs}$ after the overlap between the optical beams, as reported in Fig. 3. The optical excitation (impinging fluence $4.4 \mathrm{~mJ} / \mathrm{cm}^{2}$ ) results into two effects: a decrease in the ellipticity $\epsilon$ associated with the free layer, while no changes are visible in the magnitude of the hysteresis associated with the pinned one. A rigid shift towards a smaller magnetic field 


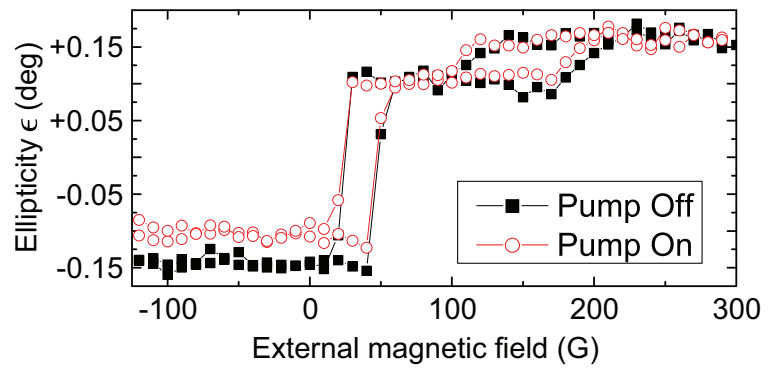

FIG. 3. (Color online) Representative Kerr ellipticity $\epsilon$ for an exchange-biased tunneling junction in either the absence or the presence of optical excitation. The second case is measured at $t=300 \mathrm{fs}$ after the overlap, at the maximum demagnetization. The reduction in the ellipticity contribution from the top layer is apparent. A small reduction in the pinning strength is also visible in the ellipticity contribution from the pinned layer. This change is smaller than $3 \%$ at an impinging fluence of $4.4 \mathrm{~mJ} / \mathrm{cm}^{2}$. The curves are measured on a device with diameter $d=125 \mu \mathrm{m}$.

is also present in this latter contribution, meaning that the increase in temperature due to the optical excitation may indeed reduce the pinning strength of the IrMn layer. This effect is smaller than $3 \%$, therefore it cannot explain the measured bias dependence of the optical demagnetization. It should be, however, considered for a correct quantitative estimation of the hot-electrons contribution to the demagnetization.

Therefore we can safely assume that the measured changes in ellipticity are directly proportional to the pump-induced demagnetization of the layers. But how could one explain the different trends of $\Delta \epsilon_{\max }^{\mathrm{top}} / \epsilon(0)$ and $\Delta \epsilon_{\max }^{\text {total }} / \epsilon(0)$ [Fig. 2(b)]? If ultrafast demagnetization in multilayered metals can be solely explained by thermal excitation and/or spin-flip events due to scattering, then it should not be possible to see any dependence on the bias voltage. We can thus conclude that the bias dependence demonstrates that part of the demagnetization can be attributed to spin-polarized currents, promoted by the optical pulse. In our experiment, the electrons are excited with relatively high energy photons $(\hbar v=3 \mathrm{eV})$, creating a significant temperature difference between the layers and a decrease in the magnetization. This leads to two effects: (i) demagnetization of thermal/scattering origin and (ii) optically excited transport of spin-polarized carriers across the MTJ barrier.

As demonstrated by Melnikov and co-workers, by optically pumping a ferromagnetic material $(\mathrm{Fe})$ a spin-polarized current is generated which can diffuse into an adjacent gold layer [29]. Similarly, we can excite a spin-polarized electron population in the free $\mathrm{CoFeB}$ layer, which can tunnel through the $\mathrm{MgO}$ barrier. In the case of Fig. 2(a), by exploiting the spin filtering effect of the MTJ (associated with the difference between the two states of conductivity $\sigma_{\uparrow \uparrow}$ and $\sigma_{\downarrow \uparrow}$ ), we achieve an intrinsic spin sensitivity for the contribution of spin-polarized currents to the demagnetization. Because mainly majority spins can flow through the device, this results in an increased degree of polarization of the photoinduced current. Minority spins have lower tunneling probability due to the low density of states available, leading to an enhancement of the measured demagnetization effect. On the other hand, by applying a voltage to the MTJ, we reduce the TMR value; this implies that it is easier for both types of electrons to cross the tunneling barrier, therefore a weaker net depletion of majority spins in the irradiated area occurs, i.e., a smaller demagnetization. The voltage-dependent reduction of $27 \%$ in $\Delta \epsilon^{\text {top }} / \epsilon(0)$ indeed corresponds remarkably well with the change in TMR of about $30 \%$ shown in Fig. 1(b). According to this analysis, we actually do not expect any $\Delta \epsilon^{\text {total }} / \epsilon(0)$ dependence on $V_{\text {bias }}$ [red open squares in Fig. 2(b)] since, in this case, we are comparing two measurements with equal conductivity [Eqs. (1)], thus the spin dependence is lost.

In conclusion, we have demonstrated that transport phenomena may be responsible for a sizable amount (up to $40 \%$ ) of ultrafast femtosecond laser-induced demagnetization in MTJ devices. We can consequently modulate the laserinduced ultrafast demagnetization in a MTJ with a bias voltage applied to the structure. We can explain this behavior in terms of optically excited spin-polarized currents through the multilayer structure.

These results may open new possibilities to control the magnetization in MTJ-based devices. For instance, assuming the reported bias-modulated demagnetization effects could be extended to ultrafast optically induced magnetization reversal, we might possibly illuminate large MTJ arrays with a single laser pulse and electrically address only selected devices. One might thus obtain a faster version of heat-assisted solid-state memories [14,34] where the size of the memory cells would not be limited by diffraction, with the possibility of operating massively parallel memory switching.

We thank M. Battiato for stimulating discussions. This research has received funding from Stichting voor Fundamenteel Onderzoek der Materie (FOM), De Nederlandse Organisatie voor Wetenschappelijk Onderzoek (NWO), the European Union (EU) Nano Sci-European Research Associates (ERA) project FENOMENA, ERC Grant Agreement No. 257280 (Femtomagnetism), and EC FP7 Grant No. 281043 (FEMTOSPIN).
[1] B. Dieny, R. Sousa, J. Herault, C. Papusoi, G. Prenat, U. Ebels, D. Houssameddine, B. Rodmacq, S. Auffret, L. Buda-Prejbeanu, M. Cyrille, B. Delaet, O. Redon, C. Ducruet, J.-P. Nozieres, and I. Prejbeanu, Int. J. Nanotechnol. 7, 591 (2010).

[2] G.-X. Miao, M. Munzenberg, and J. Moodera, Rep. Prog. Phys. 74, 036501 (2011).
[3] E. Beaurepaire, J.-C. Merle, A. Daunois, and J.-Y. Bigot, Phys. Rev. Lett. 76, 4250 (1996).

[4] A. Kirilyuk, A. V. Kimel, and T. Rasing, Rev. Mod. Phys. 82, 2731 (2010).

[5] P. S. Keatley, V. V. Kruglyak, P. Gangmei, and R. J. Hicken, Proc. R. Soc. A 369, 3115 (2011). 
[6] M. Krupa and M. Korostil, Int. J. Mod. Phys. B 26, 1250177 (2012).

[7] K. Uchida, S. Takahashi, K. Harii, J. Ieda, W. Koshibae, K. Ando, S. Maekawa, and E. Saitoh, Nature (London) 455, 778 (2008).

[8] M. Walter, J. Walowski, V. Zbarsky, M. Munzenberg, M. Schaefers, D. Ebke, G. Reiss, A. Thomas, M. Peretzki, P. Seibt, J. S. Moodera, M. Czerner, M. Bachmann, and C. Heiliger, Nat. Mater. 10, 742 (2011).

[9] H. Nakayama, M. Althammer, Y.-T. Chen, K. Uchida, Y. Kajiwara, D. Kikuchi, T. Ohtani, S. Geprägs, M. Opel, S. Takahashi, R. Gross, G. E. W. Bauer, S. T. B. Goennenwein, and E. Saitoh, Phys. Rev. Lett. 110, 206601 (2013).

[10] G. Woltersdorf, O. Mosendz, B. Heinrich, and C. H. Back, Phys. Rev. Lett. 99, 246603 (2007).

[11] G. Malinowski, F. D. Longa, J. H. H. Rietjens, P. V. Paluskar, R. Huijink, H. J. M. Swagten, and B. Koopmans, Nat. Phys. 4, 855 (2008).

[12] E. Turgut, C. La-o-vorakiat, J. M. Shaw, P. Grychtol, H. T. Nembach, D. Rudolf, R. Adam, M. Aeschlimann, C. M. Schneider, T. J. Silva, M. M. Murnane, H. C. Kapteyn, and S. Mathias, Phys. Rev. Lett. 110, 197201 (2013).

[13] M. Battiato, K. Carva, and P. M. Oppeneer, Phys. Rev. Lett. 105, 027203 (2010).

[14] I. L. Prejbeanu, M. Kerekes, R. C. Sousa, H. Sibuet, O. Redon, B. Dieny, and J. P. Nozières, J. Phys.: Condens. Matter 19, 165218 (2007).

[15] T. Aoki, Y. Ando, D. Watanabe, M. Oogane, and T. Miyazaki, J. Appl. Phys. 103, 103911 (2008).

[16] See Supplemental Material at http://link.aps.org/supplemental/ 10.1103/PhysRevB.89.140402 for further details.

[17] X. Kozina, S. Ouardi, B. Balke, G. Stryganyuk, G. H. Fecher, C. Felser, S. Ikeda, H. Ohno, and E. Ikenaga, Appl. Phys. Lett. 96, 072105 (2010).

[18] E. Albisetti, D. Petti, M. Cantoni, F. Damin, A. Torti, M. Chiari, and R. Bertacco, Biosens. Bioelectron. 47, 213 (2013).
[19] D. Djayaprawira, K. Tsunekawa, M. Negai, H. Maehara, S. Yamagata, N. Watanabe, S. Yuasa, Y. Suzuki, and K. Ando, Appl. Phys. Lett. 86, 092502 (2005).

[20] C.-Y. You and S.-C. Shin, Appl. Phys. Lett. 69, 1315 (1996).

[21] J. Bernos, M. Hehn, F. Montaigne, C. Tiusan, D. Lacour, M. Alnot, B. Negulescu, G. Lengaigne, E. Snoeck, and F. G. Aliev, Phys. Rev. B 82, 060405 (2010).

[22] E. Carpene, E. Mancini, C. Dallera, M. Brenna, E. Puppin, and S. De Silvestri, Phys. Rev. B 78, 174422 (2008).

[23] M. Krauß, T. Roth, S. Alebrand, D. Steil, M. Cinchetti, M. Aeschlimann, and H. C. Schneider, Phys. Rev. B 80, 180407 (2009).

[24] U. Atxitia, O. Chubykalo-Fesenko, J. Walowski, A. Mann, and M. Münzenberg, Phys. Rev. B 81, 174401 (2010).

[25] G. P. Zhang and W. Hübner, Phys. Rev. Lett. 85, 3025 (2000).

[26] B. Koopmans, G. Malinowski, F. Della Longa, D. Steiauf, M. Fhnle, T. Roth, M. Cinchetti, and M. Aeschlimann, Nat. Mater. 9, 259265 (2010).

[27] J.-Y. Bigot and E. Beaurepaire, Nat. Phys. 5, 515 (2009).

[28] P. M. Oppeneer and A. Liebsch, J. Phys.: Condens. Matter 16, 5519 (2004).

[29] A. Melnikov, I. Razdolski, T. O. Wehling, E. T. Papaioannou, V. Roddatis, P. Fumagalli, O. Aktsipetrov, A. I. Lichtenstein, and U. Bovensiepen, Phys. Rev. Lett. 107, 076601 (2011).

[30] E. Carpene, F. Boschini, H. Hedayat, C. Piovera, C. Dallera, E. Puppin, M. Mansurova, M. Münzenberg, X. Zhang, and A. Gupta, Phys. Rev. B 87, 174437 (2013).

[31] W.-G. Wang, M. Li, S. Hageman, and C. L. Chien, Nat. Mater. 11, 64 (2012).

[32] T. Nozaki, Y. Shiota, M. Shiraishi, T. Shinjo, and Y. Suzuki, Appl. Phys. Lett. 96, 022506 (2010)

[33] J. Kim, J. Sinha, M. Hayashi, M. Yamanouchi, S. Fukami, T. Suzuki, S. Mitani, and H. Ohno, Nat. Mater. 12, 240 (2013).

[34] G. D. Fuchs, N. C. Emley, I. N. Krivorotov, P. M. Braganca, E. M. Ryan, S. I. Kiselev, J. C. Sankey, D. C. Ralph, R. A. Buhrman, and J. A. Katine, Appl. Phys. Lett. 85, 1205 (2004). 